\title{
Estratégia alimentar com ciclos de restrição e realimentação no desempenho produtivo de juvenis de tilápia do Nilo da linhagem GIFT
}

\author{
Feeding strategies with cycles of restriction and refeeding on growth performance of Nile tilapia \\ juveniles, GIFT line
}

\author{
Eduardo Henrique da Palma ${ }^{\mathrm{I}}$ Leonardo Susumu Takahashi ${ }^{{ }^{*}}$ Luciana Thie Seki Dias $^{\mathrm{II}}$ \\ Rodrigo Yukihiro Gimbo ${ }^{I}$ Juliana Tomomi Kojima ${ }^{I}$ Daniel Nicodemo $^{\mathrm{I}}$
}

RESUMO

O objetivo deste estudo foi avaliar a ocorrência de resposta compensatória no desempenho produtivo de juvenis de tilápia do Nilo Oreochromis niloticus, linhagem GIFT, submetidos a diferentes estratégias alimentares. Foram utilizados 135 juvenis de tilápia, distribuídos em nove tanques de polietileno de $100 \mathrm{~L}$ cada. As estratégias testadas foram: grupo controle (alimentado todo dia), grupo alimentado por cinco dias seguidos de dois dias de restrição de alimento (5A) $2 R)$ e grupo alimentado por quatro dias seguidos de três dias de restrição de alimento (4A/3R). Foram avaliados parâmetros físico-químicos da água e de desempenho produtivo. Os resultados foram submetidos à análise de variância, e as médias foram comparadas pelo teste Tukey, a $5 \%$ de probabilidade. A qualidade de água, o fator de condição e a conversão alimentar não foram influenciados pela estratégia alimentar. O grupo alimentado com a estratégia $5 A / 2 R$ apresentou peso final, ganho de peso e taxa de crescimento específico semelhantes ao grupo continuamente alimentado (7,8 e 9,2g; 6,4 e 7,8g e 2,7 e 3,0\% dia ${ }^{-1}$, para peso final, ganho de peso e taxa de crescimento especifico, respectivamente). A estratégia 4A/3R apresentou os piores resultados de desempenho produtivo, e a estratégia $5 A / 2 R$ pode ser usada na alimentação de juvenis de tilápia do Nilo sem prejuízo ao desempenho produtivo, possibilitando inclusive redução de até $22,5 \%$ na quantidade de alimento ofertada.

Palavras-chave: privação alimentar, estratégia alimentar, Oreochromis niloticus.

\section{ABSTRACT}

The objective of this study was to evaluate the occurrence of compensatory answer on productive performance of Nile tilapia Oreochromis niloticus juveniles, GIFT line, submitted to different feeding strategies. It was used 135 juveniles tilapias distributed in nine $100 \mathrm{~L}$ polyethylene tanks each. The tested strategies were the control group (fed everyday), group fed for 5 days followed by 2 days of food restriction ( $5 \mathrm{~A}$ $2 R$ ) and group fed for 4 days followed by 3 days of food restriction $(4 A / 3 R)$. Were evaluated physical chemical water parameters e growth performance. Results were submitted to an analysis of variance and averages compared by Tukey test $5 \%$. Water quality, condition factor and feed conversion were not influenced by feeding strategy. Group fed 5A/2R strategy showed final weight, weight gain and specific growth rate similar to group continually fed $(7.8$ e $9.2 \mathrm{~g} ; 6.4$ e $7.8 \mathrm{~g}$ e 2.7 e $3.0 \%$ $d^{2} y^{-1}$, for final weight, weight gain and specific growth rate, respectively). The strategy $4 A / 3 R$ showed the poorest productive performance. Strategy $5 A / 2 R$ can be used in Nile tilapia juveniles feeding without damage to the fish productive performance, allowing a reduction of up to $22.5 \%$ on the feed quantity offered.

Key words: food deprivation, feeding strategy, Oreochromis niloticus.

\section{INTRODUÇÃO}

Em razão da expansão da aquicultura e da demanda de pescado pela população mundial, o estudo e desenvolvimento de pacotes tecnológicos específicos para diferentes espécies de organismos aquáticos se tornam primordiais para a competitividade no mercado atual. Pelo fato de a nutrição e alimentação representarem cerca de $70 \%$ do custo de produção, estratégias básicas para o aperfeiçoamento na utilização dos recursos podem ser aplicadas na atividade com o intuito de aumentar a produtividade e qualidade do produto final. A adoção do manejo alimentar ideal, nas

IFaculdade de Zootecnia, Campus de Dracena, Universidade Estadual Paulista (UNESP), 17900-000, Dracena, SP, Brasil. E-mail: takahashi@dracena.unesp.br. *Autor para correspondência.

IICentro de Ciências Agrárias, Universidade Federal de São Carlos (UFSCAR), Araras, SP, Brasil. 
diferentes fases de desenvolvimento dos peixes, permite melhorar o seu crescimento, sua sobrevivência e sua conversão alimentar, contribuindo para reduzir o desperdício de ração (KINDSCHI, 1998).

Como forma de adaptação a variações na ingestão de alimento decorrentes da escassez temporal e espacial ou em razão da migração para a desova, muitos organismos exibem respostas compensatórias. O crescimento compensatório pode ser definido como um processo fisiológico por meio do qual um organismo tem seu crescimento acelerado após um período de desenvolvimento reduzido, geralmente provocado pela diminuição na ingestão de alimento, na tentativa de alcançar o peso dos animais que cresceram continuamente (HORNICK et al., 2000).

O crescimento compensatório pode ser dividido em categorias de acordo com sua extensão. Pode-se citar a compensação parcial, compensação completa e sobre compensação. No primeiro caso, após um período, os animais submetidos à restrição alimentar e realimentados não alcançam o porte de animais da mesma idade sem restrição alimentar, mas apresentam alta ingestão de alimento, crescimento e melhor conversão alimentar. Já no segundo caso, os animais submetidos à restrição alimentar e realimentados atingem o mesmo porte dos animais de mesma idade que receberam alimentação contínua. No terceiro caso, os animais submetidos à restrição alimentar e realimentados são maiores que os animais que não sofreram restrição (ALI et al., 2003).

A importância do ganho de peso compensatório depende de alguns fatores, tais como : a natureza, severidade e duração da restrição alimentar, o estágio de desenvolvimento dos peixes em que se inicia a subnutrição, a idade de maturidade sexual e o modelo de realimentação (WANG et al., 2000; ALI et al., 2003; TIAN \& QIN, 2003). A maioria dos estudos sobre crescimento compensatório em peixes tem utilizado períodos longos de jejum, sendo dada pouca atenção ao efeito da restrição alimentar por períodos mais curtos. Segundo KINDSCHI (1988), o ciclo semanal de arraçoamento, incluindo um ou dois dias de jejum, pode compensar pela redução dos custos com ração e mão-de-obra. Além disso, tanto estratégias de alimentação com apenas uma fase de restrição alimentar e realimentação, como vários ciclos de restrição e realimentação, têm sido usadas para promover respostas compensatórias no desempenho produtivo de peixes.

Juvenis de tilápia do Nilo submetidos a ciclos de um dia de jejum por semana apresentaram um crescimento mais rápido em comparação com os peixes diariamente alimentados, durante 24 semanas, a $27^{\circ} \mathrm{C}$
(MÉLARD et al., 1997). Por outro lado, em tilápia híbrida O. mossambicus $x \boldsymbol{O}$. niloticus, após quatro semanas de realimentação, o peso corporal dos peixes submetidos à restrição alimentar por período de duas a quatro semanas foi menor do que o do grupo controle, apesar da ocorrência de hiperfagia (WANG et al., 2000).

O crescimento compensatório em peixes pode ter importante aplicação na aquicultura. Uma exploração adequada desse fenômeno pode resultar em melhor produção, com aumento na taxa de crescimento e eficiência alimentar e redução do custo com alimentação (HAYWARD et al., 1997; WANG et al., 2000; MACLEAN \& METCALFE, 2001). O objetivo deste trabalho foi avaliar a ocorrência de resposta compensatória e o desempenho produtivo de juvenis de tilápia do Nilo, linhagem GIFT, submetidos a diferentes estratégias alimentares.

\section{MATERIAL E MÉTODOS}

O experimento foi conduzindo na Faculdade de Zootecnia, da Universidade Estadual Paulista (UNESP), campus de Dracena, no período de 14 de março a 15 de maio de 2008. Foram utilizados nove tanques de polietileno, com capacidade para 100L cada, dispostos num sistema fechado com circulação contínua de água, filtragem biológica para retirada da amônia tóxica e iluminação natural. A cada três dias, por sifonamento, foi retirada a matéria orgânica depositada no fundo dos tanques, com reposição da água perdida. Durante o experimento, foram monitorados diariamente os seguintes parâmetros físico-químicos da água: temperatura da água, concentração de oxigênio dissolvido (medidor eletrônico portátil F-HI9147, Hanna) e pH (pHmetro digital portátil pHteck), e a cada dois dias foram determinadas as concentrações de amônia total (metodologia colorimétrica, com Reativo de Nessler).

Foram utilizados 135 juvenis de tilápia do Nilo, linhagem GIFT, revertidos sexualmente para macho e provenientes da piscicultura Aracanguá. Inicialmente esses animais foram mantidos nos tanques de polietileno de 100L por um período de sete dias para aclimatação, sendo alimentados duas vezes ao dia com ração extrusada comercial (32\% PB), até a saciedade aparente dos peixes. Após a aclimatação, os animais foram submetidos a uma biometria inicial, com uso do anestésico benzocaína (100mg $\mathrm{L}^{-1}$ de água), para avaliação do peso inicial $(1,37 \pm 0,01 \mathrm{~g})$ e comprimento total inicial $(4,09 \pm 0,07 \mathrm{~cm})$. Em seguida, foram distribuídos aleatoriamente nos nove tanques de polietileno, na densidade de 15 animais por tanque. 
As estratégias de alimentação avaliadas foram: peixes alimentados continuamente (controle), peixes alimentados em ciclos compostos por cinco dias de alimentação seguidos de dois dias de restrição de alimento $(5 \mathrm{~A} / 2 \mathrm{R})$ e peixes alimentados em ciclos compostos por quatro dias de alimentação seguidos de três dias de restrição de alimento (4A/3R), correspondendo aos três tratamentos experimentais, com três repetições cada tratamento. Em todos os tratamentos foi oferecida ração extrusada comercial (onívoros crescimento) contendo $32 \% \mathrm{~PB}$, até a saciedade aparente. $\mathrm{O}$ alimento foi ofertado duas vezes ao dia (8 e 16 horas), durante o período de 63 dias de experimento. Foi realizado rigoroso controle do consumo de ração, para posteriores cálculos dos parâmetros de eficiência alimentar.

Ao final do experimento, foi realizada uma biometria final para avaliação do crescimento e ganho de peso dos peixes. Por meio dos dados obtidos na biometria inicial e final, foram calculados os seguintes parâmetros de desempenho produtivo: peso final, comprimento total final, ganho de peso, taxa de crescimento específico, conversão alimentar, consumo médio, fator de condição e consumo total de ração. O experimento foi conduzido num delineamento inteiramente casualizado, com três tratamentos (controle, 5A/2R, 4A/3R) e três repetições cada. Os resultados obtidos foram submetidos à análise de variância, e as médias foram comparadas pelo teste Tukey, a 5\% de probabilidade. As análises foram realizadas com o auxílio do programa ESTAT versão 2.0.

\section{RESULTADOS E DISCUSSÃO}

Os parâmetros de qualidade de água não apresentaram variações significativas no $\mathrm{pH}$, na temperatura, na concentração de oxigênio dissolvido e na concentração de amônia total da água (Tabela 1). Durante o experimento, os valores desses parâmetros permaneceram dentro das faixas consideradas adequadas para o bom desenvolvimento da tilápia do Nilo, que são: $\mathrm{pH}$ entre 6,5 a 8,0, temperatura entre 18 e $30^{\circ} \mathrm{C}$, concentração de oxigênio dissolvido acima de 4,0mg L ${ }^{-1}$ e concentração de amônia menor que $0,2 \mathrm{mg} \mathrm{L}^{-1}$ (KUBITZA, 2000).

Vale ressaltar que o experimento foi conduzido num sistema fechado com recirculação de água. Dessa forma, a água que saía dos tanques era canalizada para um tanque de $1000 \mathrm{~L}$, onde passava por um filtro biológico e, posteriormente, a água retornava aos tanques por meio de uma bomba de água submersa. A eficiência desse sistema justifica o fato de não terem sido observadas diferenças nos parâmetros de qualidade de água entre os diferentes tratamentos testados.

Ao final dos 63 dias de experimento, os peixes submetidos à estratégia $5 \mathrm{~A} / 2 \mathrm{R}$ apresentaram peso final e comprimento final semelhantes aos peixes continuamente alimentados. No entanto, esse resultado não foi observado na estratégia 4A/3R, que resultou em peixes com peso final e comprimento final inferiores $(\mathrm{P}<0,05)$ aos peixes continuamente alimentados (Tabela 2). No presente experimento, realizado com juvenis de tilápia do Nilo, fase de desenvolvimento dos peixes que apresenta alta taxa de crescimento, a privação alimentar por três dias a cada semana parece ter sido muito severa, resultando em limitada capacidade de crescimento compensatório e/ou perda de peso dos peixes, assim como observado em tilápia híbrida (WANG et al., 2000). Segundo METCALFE \& MONAGHAN (2001), deficiências nutricionais originadas de longos períodos de restrição de alimento em estágios iniciais de desenvolvimento podem comprometer a habilidade dos organismos de recuperarem-se quando os níveis adequados de alimentação são restabelecidos.

De forma semelhante à resposta observada para peso final, os animais submetidos à estratégia $5 \mathrm{~A} /$ $2 \mathrm{R}$ não apresentaram diferença $(\mathrm{P}>0,05)$ no ganho de peso quando comparados aos peixes continuamente alimentados (Tabela 2). As respostas observadas no

Tabela 1 - Parâmetros físico-químicos da água: pH, temperatura, concentração de oxigênio dissolvido (OD) e concentração de amônia total $\left(\mathrm{N}-\mathrm{NH}_{3}\right)$ dos tanques de juvenis de tilápia do Nilo submetidos a diferentes estratégias de alimentação ${ }^{1}$.

\begin{tabular}{lcccc}
\hline & $\mathrm{pH}$ & Temperatura & OD & $\mathrm{mg} \mathrm{L}^{-1}$ \\
Estratégias & & ${ }^{\circ} \mathrm{C}$ & $8,3 \pm 0,06$ & $\mathrm{mg} \mathrm{L}^{-1}$ \\
\hline Controle & $8,1 \pm 0,01$ & $21,4 \pm 0,04$ & $8,3 \pm 0,06$ & $0,033 \pm 0,001$ \\
5A/2R & $8,1 \pm 0,02$ & $21,5 \pm 0,18$ & $8,3 \pm 0,03$ & $0,032 \pm 0,001$ \\
4A/3R & $8,2 \pm 0,19$ & $21,4 \pm 0,06$ & & \\
\hline
\end{tabular}

${ }^{1}$ Média \pm desvio padrão. 
Tabela 2 - Parâmetros de desempenho produtivo: peso final, comprimento total final, ganho de peso (GP) e taxa de crescimento específico (TCE) de juvenis de tilápia do Nilo submetidos a diferentes estratégias de alimentação ${ }^{1}$.

\begin{tabular}{ccccc}
\hline & Peso final & Comprimento final & GP & TCE \\
Estratégias & $\mathrm{g}$ & $\mathrm{cm}$ & $\mathrm{g}$ & \\
& $9,2 \pm 0,5 \mathrm{a}$ & $8,0 \pm 0,1 \mathrm{a}$ & $7,8 \pm 0,5 \mathrm{a}$ & \\
\hline Controle & $7,8 \pm 0,8 \mathrm{a}$ & $7,5 \pm 0,4 \mathrm{ab}$ & $6,4 \pm 0,8 \mathrm{a}$ & $2,7 \pm 0,2 \mathrm{a}$ \\
$5 \mathrm{~A} / 2 \mathrm{R}$ & $5,7 \pm 0,5 \mathrm{~b}$ & $6,8 \pm 0,4 \mathrm{~b}$ & $4,4 \pm 0,6 \mathrm{~b}$ & $2,3 \pm 0,1 \mathrm{~b}$ \\
$4 \mathrm{~A} / 3 \mathrm{R}$ & & & & \\
\hline
\end{tabular}

${ }^{1}$ Média \pm desvio padrão. Valores seguidos de letras diferentes na mesma coluna indicam diferenças significativas a 5\% de probabilidade, pelo teste Tukey.

crescimento desses peixes, segundo ALI et al. (2003), caracterizaram compensação total, resposta em que os peixes submetidos à privação de alimento alcançam peso final semelhante ao dos peixes continuamente alimentados. Isso também pode ser confirmado pela taxa de crescimento específico, em que os animais do grupo $5 \mathrm{~A} / 2 \mathrm{R}$ apresentaram a mesma taxa de crescimento específico do grupo continuamente alimentado. Para a estratégia 4A/3R, os peixes não alcançaram o ganho de peso do grupo continuamente alimentado (Tabela 2).

Em alevinos de walleye Sander vitreus submetidos a protocolos de alimentação semelhantes (controle, 5A/2R e 3A/4R), foi observada compensação total no peso final dos peixes submetidos a cinco dias de alimentação seguidos de dois dias de restrição de alimento, enquanto nos animais alimentados durante três dias seguidos de quatro dias de restrição ocorreu apenas compensação parcial (ROSAUER et al., 2009).

$\mathrm{O}$ fator de condição, índice que relaciona o peso com o comprimento corporal do animal, pode diferenciar os peixes alimentados dos que foram submetidos à restrição alimentar, como foi observado em juvenis de pacu Piaractus mesopotamicus, em que os peixes submetidos a 22 dias de jejum apresentaram fator de condição inferior aos peixes continuamente alimentados (GONÇALVES, 2001). No presente trabalho, não foi encontrada nenhuma diferença $(P>0,05)$ no fator de condição entre os peixes submetidos à restrição de alimento e os continuamente alimentados (Tabela 2), assim como observado por TAKAHASHI (2007) em juvenis de pacu com a adoção de ciclos curtos de restrição-realimentação, com três dias de restrição e três dias de realimentação durante 36 dias. Em juvenis de whitefish Coregonus lavaretus, a adoção de estratégias com ciclos compostos por dois dias de restrição seguidos de cinco de alimentação e dois dias de restrição seguidos de dois de alimentação não resultaram em alterações significativas no fator de condição, o que foi atribuído pelos autores a mecanismos compensatórios (KANKANEN \& PIRHONEN, 2009).
Um mecanismo que pode levar à ocorrência de ganho compensatório é a hiperfagia, que pode ser constatada pelo aumento do consumo de alimento nos dias de realimentação (ALI et al., 2003), mas essa resposta não foi observada nos juvenis da tilápia do Nilo submetidos a ciclos curtos de restriçãorealimentação (Tabela 3). Da mesma forma, não ocorreu resposta compensatória em relação ao melhor aproveitamento do alimento. Isso pode ser verificado ao observar que não houve melhora na conversão alimentar dos peixes submetidos à restrição (Tabela 3). Contrariando o encontrado por KIM \& LOVELL (1995) em trabalhos com bagre do canal Ictalurus punctatus, no qual os animais submetidos à restrição obtiveram conversão alimentar semelhantes aos peixes continuamente alimentados.

Maior ingestão de alimento foi observada em juvenis de whitefish submetidos a ciclos de dois dias de restrição seguidos de dois de alimentação e essa hiperfagia foi menos pronunciada nos peixes submetidos a dois dias de restrição seguidos de cinco dias de alimentação. Embora a restrição não tenha resultado em melhor aproveitamento do alimento, foi observada uma relação linear entre a quantidade de alimento ingerida na ultima refeição e o volume do estômago dos peixes, sugerindo aumento da

Tabela 3 - Parâmetros de desempenho produtivo: conversão alimentar (CA), consumo médio (CM) e fator de condição (K) de juvenis de tilápia do Nilo submetidos a diferentes estratégias de alimentação ${ }^{1}$.

\begin{tabular}{lccc}
\hline Estratégias & CA & \multicolumn{1}{c}{ CM } & K \\
& \multicolumn{3}{c}{ g peixe $^{-1}$} \\
\hline Controle & $1,8 \pm 0,11$ & $13,8 \pm 1,73 a$ & $0,018 \pm 0,001$ \\
5A/2R & $1,9 \pm 0,16$ & $11,9 \pm 2,15 \mathrm{ab}$ & $0,018 \pm 0,001$ \\
4A/3R & $2,1 \pm 0,16$ & $9,1 \pm 0,42 \quad$ b & $0,018 \pm 0,002$ \\
\hline
\end{tabular}

${ }^{1}$ Média \pm desvio padrão. Valores seguidos de letras diferentes na mesma coluna indicam diferenças significativas a 5\% de probabilidade, pelo teste Tukey. 
capacidade de elasticidade do estômago dos peixes submetidos à restrição (KANKANEN \& PIRHONEN, 2009).

Na observação da tabela 2, verifica-se que, para peso final e comprimento final, a estratégia $5 \mathrm{~A} / 2 \mathrm{R}$ não apresentou diferenças $(\mathrm{P}<0,05)$ em relação ao grupo continuamente alimentado, portanto ambos obtiveram desempenhos parecidos. Isso também pode ser constatado no ganho de peso e na taxa de crescimento específico. No entanto, o consumo foi maior no grupo continuamente alimentado em relação ao que recebeu a estratégia 5A/2R (Tabela 4).

Embora não tenha sido objetivo deste trabalho avaliar a redução dos custos de produção, um produtor optando pela estratégia $5 \mathrm{~A} / 2 \mathrm{R}$ poderá ter uma redução na quantidade de ração ofertada. Essa redução pode ser de 22,5\%, valores próximos aos encontrados por ODA et al. (2004), que foi de $25 \%$ em estudos com juvenis de pacu submetidos a seis dias de alimentação e um de jejum, ou ainda três dias de alimentação e um de jejum. Apesar de apresentar uma redução na quantidade de ração de $36,5 \%$, o tratamento 4A/3R não se mostrou eficaz, pois, ao final dos 63 dias, os peixes não alcançaram o peso final do grupo continuamente alimentado.

Resultados semelhantes foram observados em juvenis de tilápia híbrida Oreochromis niloticusx $\boldsymbol{O}$. aureus, nos quais a restrição de alimento durante um dia por semana resultou em peso final, ganho de peso e taxa de crescimento específico iguais aos parâmetros do grupo continuamente alimentado, mas o aumento nos dias de restrição ocasionou piora nesses parâmetros. A restrição por três dias resultou em aumento na ingestão, mas pior conversão alimentar e ganho de peso, consequentemente, pior eficiência econômica (ABDEL-HAKIM et al., 2009).

Em outro estudo, juvenis de tilápia do Nilo submetidos a ciclos compostos por uma semana de

Tabela 4 - Consumo total de ração (CTR) e redução da quantidade de ração oferecida a juvenis de tilápia do Nilo submetidos a diferentes estratégias de alimentação ${ }^{1}$.

\begin{tabular}{lcc}
\hline Tratamento & CTR $^{1}$ & $\begin{array}{c}\text { Redução da quantidade de } \\
\text { alimento }\end{array}$ \\
& g tratamento $^{-1}$ & $\%$ \\
\hline Controle & $186,2 \pm 1,8 \mathrm{a}$ & 0 \\
5A/2R & $144,8 \pm 9,0 \mathrm{~b}$ & 22,54 \\
4A/3R & $118,1 \pm 6,5$ c & 36,57 \\
\hline
\end{tabular}

${ }^{1}$ Média \pm desvio padrão. Valores seguidos de letras diferentes na mesma coluna indicam diferenças significativas a $5 \%$ de probabilidade, pelo teste Tukey. restrição e duas de realimentação, duas semanas de restrição e quatro de realimentação e quatro semanas de restrição e oito de realimentação apresentaram elevada ingestão de alimento e taxa de crescimento específico, mas apenas compensação parcial foi alcançada (WANG et al., 2009). Nos juvenis de tilápia do Nilo deste experimento, estratégias de alimentação com períodos de restrição superiores a dois dias não permitiram compensação total no peso final dos peixes, sugerindo ser severa para os peixes nas condições experimentais avaliadas.

\section{CONCLUSÃO}

De acordo com os resultados do presente estudo, realizado com juvenis da tilápia do Nilo Oreochromis niloticus, sugere-se a estratégia 5A/2R como ferramenta de redução na quantidade de ração ofertada sem prejuízo ao desempenho produtivo dos peixes. No entanto, estudos com avaliação econômica dessas estratégias devem ser realizados.

\section{COMITÊ DE ÉTICA E BIOSSEGURANÇA}

Este trabalho foi realizado de acordo com as normas éticas, sendo aprovado (certificado número 006/2008) pela Comissão de Ética em Experimentação Animal (CEEA) da Faculdade de Zootecnia de Dracena - UNESP.

\section{REFERÊNCIAS}

ABDEL-HAKIM et al. Effect of feeding regimes on growth performance of juvenile hybrid tilapia (Oreochromis niloticusxOreochromis aureus). World J Agric Sci, v.5, n.1, p.49-54, 2009. Disponível em: <http://www.idosi.org/wjas/ wjas5(1)/7.pdf>. Acesso em: 22 maio 2009.

ALI, M. et al. Compensatory growth in fishes: a response to growth depression. Fish Fish, v.4, n.2, p.147-190, 2003. Disponível em: <http://www.uniovi.es/nicieza/publs/FF4.pdf>. Acesso em: 18 ago. 2008 .

GONÇALVES, F.D. Metabolismo energértico e desempenho produtivo de juvenis de pacu (Piaractus mesopotamicus), submetidos a jejum e realimentação com dietas contendo diferentes níveis de carboidratos e proteína. 2001. 671f. Dissertação (Mestrado em Aquicultura) - Centro de Aqüicultura, Universidade Estadual Paulista, Jaboticabal, SP.

HAYWARD, R.S. et al. Use of compensatory growth to double hybrid sunfish growth rates. T Am Fish Soc, v.126, n.2, p.316322, 1997. Disponível em: <http://afsjournals.org/doi/abs/10.1577/ 1548-8659(1997)126\%3C0316:NUOCGT\%3E2.3.CO\%3B2>. Acesso em: 18 ago. 2008. doi: 10.1577/15488659(1997)126<0316:NUOCGT>2.3.CO;2.

HORNICK, J.L. et al. Mechanisms of reduced and compensatory growth. Domest Anim Endocrin, v.19, n.2, p.121-132, 2000. Disponível em: <http://www.sciencedirect.com/ 
science?_ob=MImg\&_imagekey=B6T62-41C2SFN-6J \&_c di $=5018$ \&_us e r $=972052 \&$ \&_orig = s e a r c h \&_c over Date $=08 \% 2$ F $31 \% 2$ F $2000 \&$ \& s k $=$ $999809997 \& \mathrm{vi}$ e w $=\mathrm{c} \& \mathrm{wch}$ p $=\mathrm{d}$ G L b V t b zSkzk\&md5=85e672ddcc0c2f54862ffdef4edc83c1\&ie =/ sdarticle.pdf $>$. Acesso em: 18 ago. 2008. doi: 10.1016/S07397240(00)00072-2.

KANKANEN, M.; PIRHONEN, J. The effect of intermittent feeding on feed intake and compensatory growth of whitefish Coregonus lavaretus L. Aquaculture, v.288, p.92-97, 2009. Disponível em: $<$ h t t p : / / w w w. s c i e n c ed i r e c t. c o m / science?_ob=MImg\&_imagekey=B6T4D-4V17CKG-1-

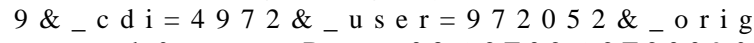
=search \&_cover Date $=03 \% 2$ F $02 \% 2$ F $2009 \&$ _ s k = $997119998 \&$ \& i e w = c \& w c h p = d G L b V l z zSkWb\&md5=67378fe6a9a63797bf7148b1daa2075a\&ie=/ sdarticle.pdf $>$. Acesso em: 22 maio 2009. doi:10.1016/ j.aquaculture.2008.11.029.

KIM, M.K.; LOVELL, R.T. Effect of restricted feeding regimes on compensatory weigth gain and body tissue changes in channel catfish (Ictalurus punctatus) in ponds. Aquaculture, v.135, n.4, p.285-293, 1995. Disponível em: <http://www.sciencedirect.com/ science?_ob=MImg\&_imagekey=B6T4D-3Y45G2S-7V$2 \&$ \& d i $=4972$ \&_user $=972052$ \&_orig = se ar ch \&_c overDate=10\%2F15\%2F1995\&_sk=998649995\&view=c\&wchp=dGLzVzzzSkWA\&md5=f2b1eb05117b44b071ff70703c0339a8\&ie=/ sdarticle.pdf>. Acesso em: 18 ago. 2008. doi:10.1016/00448486(95)01027-0.

KINDSCHI, G.A. Effect of intermittent feeding on growth of rainbow trout (Salmo gairdneri) Richardson. Aquacult Fish Manage, v.19, n.2, p.213-215, 1988.

KUBITZA, F. Tilápia: tecnologia e planejamento na produção comercial. Jundiaí: F. Kubitza, 2000. 285p.

MACLEAN, A.; METCALFE, N.B. Social status, access to food, and compensatory growth in juvenile Atlantic salmon. $\mathbf{J}$ Fish Biol, v.58, n.5, p.1331-1346, 2001. Disponível em: <http://www3.interscience.wiley.com/cgi-bin/fulltext/ 118974456/PDFSTART>. Acesso em: 20 ago. 2008. doi: 10.1006/jfbi.2000.1545.

MÉLARD, C. et al. Compensatory growth of Nile tilapia Oreochromis niloticus. In: INTERNATIONAL SYMPOSIUM ON TILAPIA IN AQUACULTURE 4., 1997, Orlando. Proceedings... Orlando: NRAES, 1997. V.1, p.178-185.

METCALFE, N.B.; MONAGHAN, P. Compensation for bad start: grow now, pay later? Trends Ecol Evol, v.16, n.5, p.254260, 2001. Disponível em: <http://www.sciencedirect.com/ science?_ob=MImg\&_imagekey=B6VJ1-42RMMHV-T-

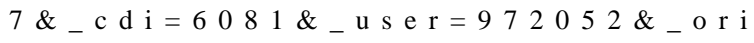
$\mathrm{g}=$ s e a r c h \&_cover Dat e $=05 \% 2$ F $01 \% 2$ F 200 $1 \&$ \& s $=999839994 \&$ vie w $=$ c \& w chp $=$ d G L b V l WzSkzk\&md5 $=4175$ c4858ca25cdc0fd76eaa3a21f382\&ie $=$ / sdarticle.pdf>. Acesso em: 16 ago. 2008. doi:10.1016/S01695347(01)02124-3.

ODA, G.M. et al. Desempenho produtivo de juvenis de pacu (Piaractus mesopotamicus), submetidos a diferentes estratégias de alimentação. In: AQUACIÊNCIA 2004 - CONGRESSO DA SOCIEDADE BRASILEIRA DE AQÜICULUTURA E BIOLOGIA AQUÁTICA, 2004, Vitória, ES. Anais... Vitória: Aquabio, 2004. p.93.

ROSAUER, D.R. et al. Role of compensatory growth in walleye fingerling production. North Am J Aquac, v.71, p.35-38, 2009. Disponível em: <http://afsjournals.org/doi/pdf/10.1577/ A07-064.1>. Acesso em: 22 maio 2009. doi: 10.1577/A07064.1 .

TAKAHASHI, L.S. Estratégia alimentar, teores de carboidratos dietéticos, desempenho e respostas fisiológicas do pacu (Piaractus mesopotamicus). 2007. $89 \mathrm{f}$. Tese (doutorado em Zootecnia) - Faculdade de Ciências Agrárias e Veterinárias, Universidade Estadual Paulista, Jaboticabal, SP.

TIAN, X.; QIN, J.G. A single phase of food deprivation provoked compensatory growth in barramundi (Lates calcarifer). Aquaculture, v.224, n.1-4, p.169-179, 2003. Disponível em: <http://www.sciencedirect.com/ science?_ob=MImg\&_imagekey=B6T4D-48BTY79-1$9 \&$ \& d i $=4972 \&$ \&_us e r $=972052 \&$ \& or ig

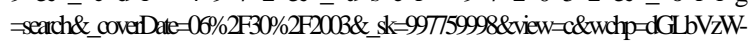
zSkWA\& $m d 5=c 821$ df6dddaa0dd751dbdfc8e20b9561\&ie = / sdarticle.pdf>. Acesso em: 18 ago. 2008. doi: 10.1016/S00448486(03)00224-2.

WANG, Y. et al. Compensatory growth in hybrid tilapia (Oreochromis mossambicusxO. niloticus), reared in sea water. Aquaculture, v.189, n.1-2, p.101-108, 2000. Disponível em: $<$ h t t p : / / w w w. s c i e n c ed i r e c t. c o m/ science?_ob=MImg\&_imagekey=B6T4D-40HV08M-9$1 \&$ \&_di $=4972 \&$ \& user $=972052 \&$ \&orig $=$ search\&_c overDate=09\%2F25\%2F2000\&_sk=998109998\&view=c\&wchp=dGLbVlzzSkzS\&md5=3def80661cf95c43d0e074393f107d8a\&ie=/ sdarticle.pdf>. Acesso em: 16 ago. 2008. doi: 10.1016/S00448486(00)00353-7.

WANG, Y. et al. Cyclical feed deprivation and refeeding fails to enhance compensatory growth in Nile tilapia, Oreochromis niloticus L. Aquac Res, v.40, p.204-210, 2009. Disponível em: <http://www3.interscience.wiley.com/cgi-bin/fulltext/ 121378392/PDFSTART $>$. Acesso em: 22 maio 2009. doi: 10.1111/j.1365-2109.2008.02083.x. 